\title{
Diagnostic accuracy of the HEART Pathway and EDACS-ADP when combined with a 0-hour/1-hour hs-cTnT protocol for assessment of acute chest pain patients
}

\author{
Tsvetelina Nilsson (1) , ${ }^{1}$ Erik Johannesson, ${ }^{2}$ Jakob Lundager Forberg, ${ }^{3}$ Arash Mokhtari, ${ }_{1}^{4}$ \\ UIf Ekelund ${ }^{1}$
}

Handling editor Edward Carlton

- Additional material is published online only. To view, please visit the journal online (http://dx.doi.org/10.1136/ emermed-2020-210833).

'Department of Emergency Medicine, Skåne University Hospital Lund, Lund University, Lund, Sweden

${ }^{2}$ Department of Emergency

Medicine, Lund University, Lund, Sweden

${ }^{3}$ Department of Emergency Medicine and Prehospital Care, Helsingborgs lasarett, Helsingborg, Sweden ${ }^{4}$ Department of Cardiology, Skåne University Hospital Lund, Lund, Sweden

\section{Correspondence to} Dr Tsvetelina Nilsson, Department of Emergency Medicine, Skåne University Hospital Lund, Lund, Skåne, Sweden;

drcmarkova@gmail.com

$\mathrm{AM}$ and UE contributed equally.

Received 20 October 2020 Revised 11 March 2021 Accepted 26 March 2021 Published Online First 9 April 2021

\section{Linked}

- http://dx.doi.org/10.1136/ emermed-2021-211576

Check for updates

(C) Author(s) (or their employer(s)) 2021. No commercial re-use. See rights and permissions. Published by BMJ.

To cite: Nilsson T,

Johannesson $E_{\text {, Lundage }}$

Forberg J, et al. Emerg Med J 2021:38:808-813.

\section{ABSTRACT}

Background/aim In ED chest pain patients, a

0-hour/1-hour protocol based on high sensitivity cardiac troponin T (hs-cTnT) tests combined with clinical risk stratification in diagnosing acute coronary syndrome is recommended. Two of the most promising risk stratification tools are the History, ECG, Age, Risk Factors and Troponin (HEART) and Emergency Department Assessment of Chest Pain (EDAC) scores. Few studies have assessed the diagnostic accuracy of the 0-hour/1hour hs-cTnT protocol when combined with HEART score, and none with EDACS. In ED chest pain patients, we aimed to evaluate the diagnostic accuracy of a 0 -hour/1hour hs-cTnT protocol combined the HEART Pathway, or the EDACS accelerated diagnostic pathway (EDACSADP).

Methods This was a secondary analysis of data from a prospective observational study enrolling 1167 ED chest pain patients who visited the ED at Skåne University Hospital in Lund, Sweden in the period between February 2013 and April 2014. HEART and EDAC scores were assessed together with hs-cTnT at 0 and 1 hour and compared with HEART score alone. Sensitivity, specificity, negative predictive value (NPV) and likelihood ratios were evaluated. The primary outcome was major adverse cardiac events (MACE) including unstable angina within 30 days. The secondary outcome was index visit acute myocardial infarction (AMI).

Results A total of 939 patients were included in the final analysis. When combined with 0 -hour/1-hour hs-cTnT testing, the HEART Pathway and EDACS-ADP identified $49.8 \%$ and $49.6 \%$ of the patients for rule-out, with NPVs for 30 -day MACE of $99.8 \%$ and $99.1 \%$, compared with the HEART score alone that identified $53.4 \%$ of the patients for rule-out with NPV of $99.2 \%$. The NPV for index visit AMI were 100\%, 99.8\% and $99.2 \%$, respectively.

Conclusion The combination of the HEART Pathway or the EDACS-ADP with a 0-hour/1-hour hs-cTnT protocol allows safe and early rule-out in a large proportion of ED chest pain patients.

\section{INTRODUCTION}

Chest pain is a common reason for ED visits and accounts for about $10 \%$ of all non-injury-related visits in the EDs worldwide. ${ }^{1}$ A primary goal in the rapid assessment of chest pain patients is to

\section{Key messages}

What is already known on this subject

- In ED chest pain patients, the European Society of Cardiology (ESC) recommends a 0-hour/1-hour high sensitivity cardiac troponin $\mathrm{T}$ (hs-cTnT) protocol combined with clinical risk stratification to rule-out acute coronary syndrome. Several risk stratification tools such as the History, ECG, Age, Risk Factors and Troponin (HEART) and Emergency Department Assessment of Chest Pain (EDAC) scores have been presented, but the diagnostic accuracy of these scores when combined with the ESC 0 -hour/1-hour hs-cTnT protocol is unclear.

What this study adds

- Our results indicate that the combination of a 0-hour/1-hour hs-cTnT protocol with the HEART Pathway or the EDACS-accelerated diagnostic protocol allows safe and early rule-out in a large proportion of ED chest pain patients.

exclude acute coronary syndrome (ACS; acute myocardial infarction (AMI) or unstable angina (UA)). Due to the fear of inadvertently discharging a patient with ACS, a large portion of ED chest pain patients undergo extensive testing to rule-out ACS. ${ }^{2}$ However, only a minority of these patients turn out to have ACS, ${ }^{2}$ why there is a need for strategies that can rapidly identify low-risk patients suitable for safe discharge.

The initial assessment relies primarily on the history, physical examination, the ECG and repeated blood tests for cardiac troponins. ${ }^{3}{ }^{4}$ With high sensitivity cardiac troponin T (hs-cTn), shorter blood sampling intervals are now possible. The European Society of Cardiology recommends the use of a 0-hour/1-hour hs-cTn protocol for ruling out AMI. ${ }^{5}$ According to this protocol patients with an hs-cTnT $<5 \mathrm{ng} / \mathrm{L}$ at presentation ( 0 hour) or a 0 -hour hs-cTnT $<12 \mathrm{ng} / \mathrm{L}$ with a 1 -hour change $<3 \mathrm{ng} / \mathrm{L}$ are considered 'ruled out' with a high negative predictive value (NPV). An important caveat is however that a 0 -hour hs-cTnT $<5 \mathrm{ng} / \mathrm{L}$ should only be used for rule-out if the 0 -hour sample is measured $>3$ hours after chest pain onset. 
Studies indicate that the diagnostic accuracy of this approach can be enhanced by adding clinical information such as the patient history, ECG or a low Thrombolysis in Myocardial Infarction (TIMI) score. ${ }^{6-8}$

Commonly used clinical risk scores in ED chest pain patients include the HEART score (History, ECG, Age, Risk Factors and Troponin) which uses the 0 -hour troponin value only, ${ }^{6}$ the HEART Pathway which combines a low HEART score with negative serial troponins, ${ }^{9}$ or EDACS-ADP (Emergency Department Assessment for Chest Pain Score-Accelerated Diagnostic Pathway) which combines a low EDACS score with a nonischaemic ECG and negative serial troponins. ${ }^{10}$ To our knowledge, there are no studies that have evaluated the diagnostic accuracy of EDACS-ADP when combined with 0-hour/1-hour hs-cTnT, and only one have assessed the HEART Pathway when including 0 -hour/1-hour hs-cTnT. ${ }^{6}$ It is thereby unclear whether these combinations provide a safe and effective alternative in the assessment of ED chest pain patients.

In the present study, our aim was to evaluate the diagnostic accuracy of the HEART Pathway and EDACS-ADP when combined with a 0-hour/1-hour hs-cTnT protocol, and to compare with the original HEART score alone, for ruling out major adverse cardiac events (MACE) within 30 days in ED chest pain patients.

\section{MATERIAL AND METHODS Setting and study design}

The present study was a secondary analysis of data collected in a prospective observational study, the methods of which have been described in detail in a previous publication. ${ }^{7}$ We enrolled consecutive chest pain patients from February 2013 to April 2014 during weekdays 09:00 to 21:00 that visited the ED of Skåne University Hospital, Lund, Sweden, a tertiary care centre with around $65000 \mathrm{ED}$ visits annually. We included patients 18 years and older with a primary symptom of non-traumatic chest pain consistent with ACS and with stable and relatively normal vital signs (not needing immediate intervention), and where hs-cTnT was ordered. Patients who did not speak Swedish or English, or were unable to communicate due to for example, dementia, were not enrolled in the study. Patients with ST-elevation myocardial infarction (STEMI) were excluded from the analysis. Patients with haemolysis in either the 0 -hour or the 1-hour hs-cTnT sample were also excluded, as were those with missing data precluding the calculation of the HEART score or EDACS. Patients with ongoing chest pain were not excluded.

\section{Patient and public involvement}

No patient involved.

\section{Data collection}

Clinical data, blood samples and the ED physician's assessment of the patient history and the ECG were collected by research assistants using a custom questionnaire. Each physician was approached shortly after meeting the patient and assessed the likelihood of ACS based on the patient history before receiving blood sample results. Patient history was noted as highly, moderately or slightly suspicious for ACS, in accordance with the HEART score.

Lithium heparin tubes were used to collect blood samples for hs-cTnT analysis and they were analysed with the Roche Cobas e602 (Roche Diagnostics). The assay has a limit of blank of $3 \mathrm{ng} / \mathrm{L}$, a limit of detection of $5 \mathrm{ng} / \mathrm{L}$, a limit of quantification of $6 \mathrm{ng} / \mathrm{L}$ and analytical range of 5-9999 $\mathrm{ng} / \mathrm{L}$ with a coefficient of variation $<10 \%$ at the 99 th percentile cut-off point of $14 \mathrm{ng} / \mathrm{L}{ }^{11}$ Further investigation and/or treatment were at the discretion of the responsible physician, as in routine care.

\section{Index tests}

The HEART score consists of five elements-History, ECG, Age, Risk Factors and Troponin (online supplemental appendix table 1). ${ }^{6}$ Each variable is given a score from 0 to 2 points, resulting in a total maximum score of 10 , with a score $<4$ considered as low risk. The HEART score is based on the 0 -hour troponin value only. In the HEART Pathway, ${ }^{7}$ however, a HEART score $<4$ is combined with negative serial troponin testing to identify low-risk patients (online supplemental appendix table 2).

EDACS includes age, sex, risk factors and chest pain characteristics, with variables scored individually (online supplemental appendix table 3). According to the EDACS-ADP, ${ }^{10}$ patients with an EDACS score <16, a non-ischaemic ECG, and negative serial troponins are considered low risk (online supplemental appendix table 3).

The index tests evaluated were (1) a HEART score $<4$ in combination with a 0 -hour hs-cTnT $<5 \mathrm{ng} / \mathrm{L}$, or a 0 -hour hs-cTnT $<12 \mathrm{ng} / \mathrm{L}$ with a 1 -hour change $<3 \mathrm{ng} / \mathrm{L}$ (hereafter referred to as the HEART 0-hour/1-hour Pathway), (2) an EDACS score $<16$ combined with a non-ischaemic ECG and 0 -hour/1-hour hs-cTnT as described above (hereafter called EDACS 0-hour/1-hour ADP) and (3) an original HEART score $<4$ (using only 0 -hour hs-cTnT) for comparison.

\section{Outcomes and adjudication process}

The primary outcome was MACE within 30 days. MACE was defined as an adjudicated diagnosis of AMI, UA, ventricular arrhythmia requiring intervention, third-degree AV block, cardiogenic shock, cardiac arrest or death of a cardiac or unknown cause. The secondary outcome was index visit AMI. The final diagnosis of MACE within 30 days was decided by two independent cardiologists based on all available clinical data within 60 days from the index visit, such as blood samples, ECG, echocardiography, stress test and coronary angiography. In case of disagreement, a third cardiologist reviewed the patient case. Adjudicators were unaware of the research question in the present study and were blinded to the study questionnaire which contained data for calculating HEART and EDACS, as well as the 1-hour hs-cTnT result.

AMI was diagnosed based on the universal definition requiring signs or symptoms of acute ischaemia in combination with a significant increase and/or decrease in hs-cTnT with at least one value above the 99 th percentile. ${ }^{5}$ In case of late presentation to the ED, patients with elevated hs-cTnT and no significant rise or fall could still be diagnosed with AMI, if this was deemed to be the most likely diagnosis. A UA diagnosis was made in the setting of normal or only slightly elevated hs-cTnT levels (from eg, heart or kidney failure) without a significant increase and/or decrease $^{12}$ and a history consistent with UA. This was defined as new-onset angina of Canadian Cardiovascular Society class $\geq 3$, rest angina or crescendo angina and at least one of the following: coronary stenosis $\geq 70 \%$ on angiography, a positive stress test if no angiography had been performed, or new ischaemic ECG changes in patients who did not undergo a stress test or coronary angiography.

For the remaining MACE outcomes, standard definitions were used. ${ }^{13}$ 


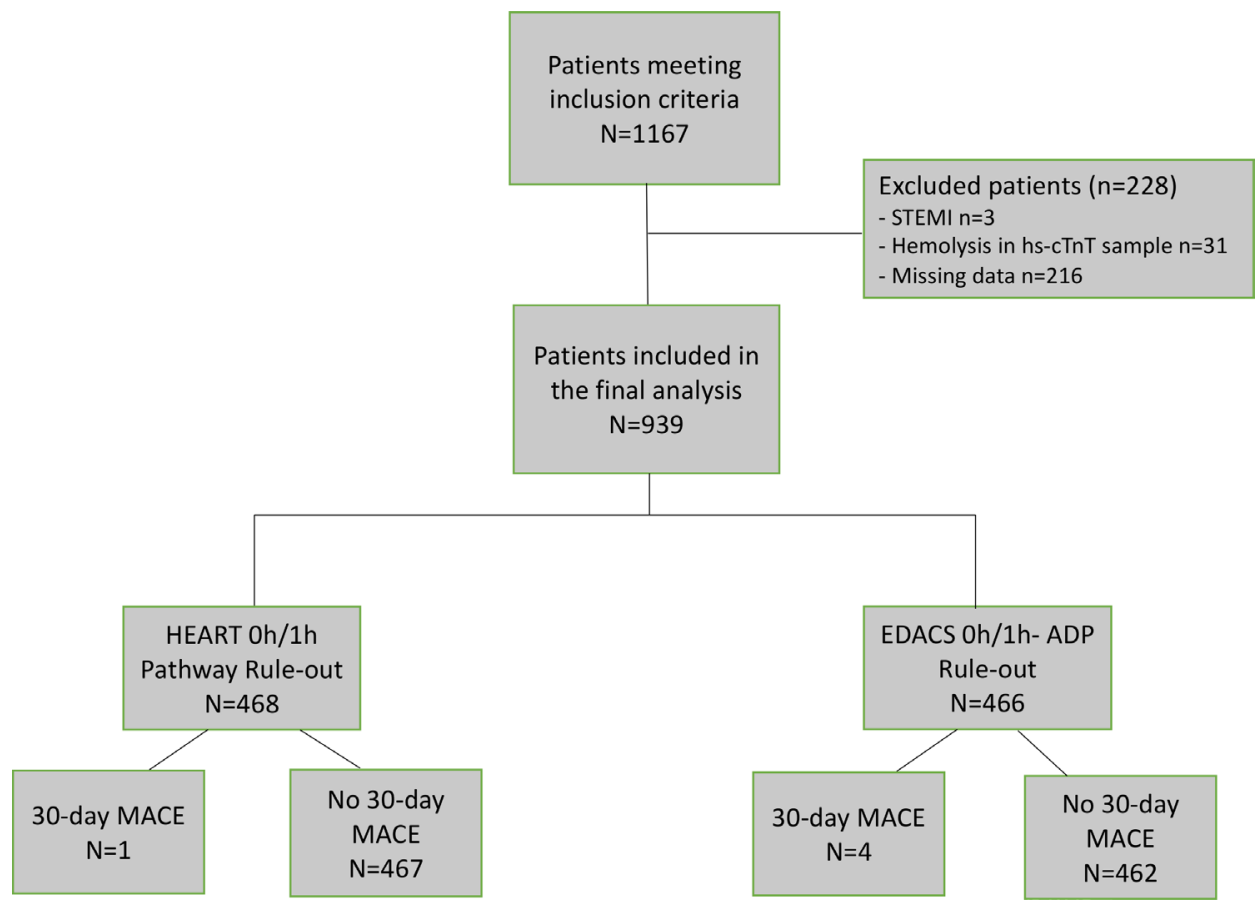

Figure 1 Patient flow chart. ADP, accelerated diagnostic pathway; EDACS, Emergency Department Assessment of Chest Pain Score; HEART, History, ECG, Age, Risk Factors and Troponin; STEMI - ST-elevation myocardial infarction, hs-CTnT, high sensitivity cardiactroponin T; MACE, major adverse cardiac events.

\section{Statistical analyses}

We used mean and SD to describe continuous variables, and proportions for categorical variables. For the primary and secondary outcomes, sensitivity, specificity, NPV and likelihood ratios (LR) and their corresponding 95\% CIs were calculated for the index tests. The $\chi^{2}$ test was used to compare differences in proportions, and the Mann-Whitney test was used to compare continuous variables.

We analysed the data with IBM SPSS V.26 (IBM) and MedCalc statistical software V.14.8.1 (MedCalc Software bvba).

\section{RESULTS}

\section{Patient characteristics}

A total of 1167 patients were eligible and enrolled in the study. As displayed in figure 1, 939 patients were included in the final analysis, including 299 patients (32.1\%) who presented to the ED within 3 hours of chest pain onset. As shown in table 1, the mean age of the included patients was 61 years, $55 \%$ were men, and $20 \%$ had a history of MI. A total of $116(12.4 \%)$ patients had a MACE within 30 days. Most of these cases were index visit AMI $(n=75 ; 64.7 \%)$ or index visit UA $(n=38 ; 32.8 \%)$.

HEART score was $<4$ in 501 patients $(53.4 \%)$, EDACS was $<16$ in 570 patients $(60.7 \%)$, and $93.3 \%$ had a non-ischaemic ECG. Of all patients, $29.7 \%$ had a 0 -hour hs-cTnT $<5 \mathrm{ng} / \mathrm{L}$, and $66.1 \%$ had a 0 -hour hs-cTnT $<12 \mathrm{ng} / \mathrm{L}$ with a 1 -hour change of $<3 \mathrm{ng} / \mathrm{L}$.

\section{Main results}

The diagnostic accuracies of the algorithms are presented in table 2 .

The HEART 0-hour/1-hour Pathway identified 468 patients (49.8\%) as low risk with an NPV of $99.8 \%$ (98.5\% to $100.0 \%)$, a sensitivity of $99.1 \%,(95.3 \%$ to $99.9 \%)$ and an LR- of 0.02 (0.00 to 0.11$)$ for 30-day MACE. This approach missed only one patient with UA. NPV for index visit AMI was thereby $100 \%$ (99.2\% to $100 \%)$ and the sensitivity $100 \%$ (95.2\% to $100.0 \%)$.

The EDACS 0-hour/1-hour ADP identified 466 patients (49.6\%) as low risk with an NPV of $99.1 \%(97.8 \%$ to $99.7 \%)$, a sensitivity of $96.7 \%$ (91.4\% to $99.1 \%)$ and an LR - of 0.06 (0.02 to 0.16$)$, missing four patients with a MACE within 30 days (one AMI, three UA). The NPV for index visit AMI was thereby $99.8 \%(98.5 \%$ to $100.0 \%)$ with a sensitivity of $98.7 \%$ (92.8\% to $100.0 \%)$.

The original HEART score identified 501 patients (53.4\%) as low risk with an NPV of $98.8 \%$ (97.4 to 99.5$)$, a sensitivity of $94.8 \%$ (89.1 to 98.1) and LR - of 0.09 (0.04 to 0.19) for 30-day MACE, missing six patients (four MI, one UA, one cardiac arrest). For index visit AMI only, the HEART score had an NPV of 99.2\% (98.0 to 99.7) and a sensitivity of $94.7 \%$ (86.9 to 98.5 ).

We also compared the performance of the HEART score or the EDACS-ADP combined with a single hs-cTnT $<5 \mathrm{ng} / \mathrm{L}$ in patients presenting $\leq 3$ hours versus $>3$ hours after chest pain onset (online supplemental appendix table 5). There were no significant differences in diagnostic performance between the two groups.

\section{DISCUSSION}

In this secondary analysis of a prospective observational study, we evaluated the diagnostic accuracy of the HEART score, the HEART Pathway and EDACS-ADP when combined with a 0-hour/1-hour hs-cTnT protocol for ruling out MACE within 30 days among ED chest pain patients. All three strategies identified a large proportion (half) of the patients as low risk with an NPV for 30-day MACE above 98\%. They thereby identified patients with an AMI or UA risk below the suggested 2\% test threshold where patients are more likely to be harmed than benefit from further testing. ${ }^{14}$ Both the HEART 0-hour/1-hour Pathway and the EDACS 0-hour/1-hour ADP also had an NPV of $\geq 99.5 \%$ for index visit AMI, which is a threshold commonly used to define what is acceptable for a chest pain rule-out strategy. ${ }^{15}$ However, 
Table 1 Patient characteristics

\begin{tabular}{|c|c|c|c|c|}
\hline & $\begin{array}{l}\text { All patients } \\
\mathrm{n}=939\end{array}$ & $\begin{array}{l}\text { HEART score } \leq 3 \\
\mathrm{n}=501\end{array}$ & $\begin{array}{l}\text { HEART 0-hour/1-hour Pathway rule-out } \\
\mathrm{n}=468\end{array}$ & $\begin{array}{l}\text { EDACS } 0 \text {-hour/1-hour ADP rule- } \\
\text { out } n=466\end{array}$ \\
\hline \multicolumn{5}{|l|}{ General information } \\
\hline Age, years mean, SD & $60.6(17.4)$ & $50.3(15.5)$ & $49.5(15.1)$ & $50.0(15.4)$ \\
\hline Arrival by ambulance & $386(41.1 \%)$ & $134(26.7 \%)$ & $119(25.4 \%)$ & $124(26.6 \%)$ \\
\hline \multicolumn{5}{|l|}{ History of } \\
\hline CABG & $81(8.6 \%)$ & $1(0.2 \%)$ & $1(0.2 \%)$ & $3(0.6 \%)$ \\
\hline $\mathrm{PCl}$ & $164(17.5 \%)$ & $18(3.6 \%)$ & $18(3.8 \%)$ & $27(5.8 \%)$ \\
\hline Diabetes mellitus type 1 or 2 & $134(14.3 \%)$ & $17(3.4 \%)$ & $16(3.4 \%)$ & $29(6.2 \%)$ \\
\hline Hypertension & $415(44.2 \%)$ & $96(19.2 \%)$ & $82(17.5 \%)$ & $108(23.2 \%)$ \\
\hline Stroke/TIA & $85(9.1 \%)$ & $2(0.4 \%)$ & $2(0.4 \%)$ & $13(2.8 \%)$ \\
\hline \multicolumn{5}{|l|}{ Current medications } \\
\hline Aspirin/ADP inhibitor & $274(29.2 \%)$ & $46(9.2 \%)$ & $41(8.8 \%)$ & $65(13.9 \%)$ \\
\hline ACE/ARB blocker & $295(31.4 \%)$ & $57(11.4 \%)$ & $49(10.5 \%)$ & $69(14.8 \%)$ \\
\hline Beta blocker & $288(30.7 \%)$ & $59(11.8 \%)$ & $50(10.7 \%)$ & $69(14.8 \%)$ \\
\hline Nitrates & $219(23.3 \%)$ & $28(5.6 \%)$ & $27(5.8 \%)$ & $44(9.4 \%)$ \\
\hline Statins & $279(29.7 \%)$ & $38(7.6 \%)$ & $35(7.5 \%)$ & $69(14.8 \%)$ \\
\hline \multicolumn{5}{|l|}{ Clinical findings } \\
\hline Mean systolic BP, mm Hg (SD) & $145(24.2)$ & $141(21.5)$ & $140(20.6)$ & $141(21.8)$ \\
\hline Mean diastolic BP, mm Hg (SD) & $85(14.1)$ & $85(13.9)$ & $85(13.5)$ & $86(13.4)$ \\
\hline ECG showing signs of acute ischaemia & $63(6.7 \%)$ & $3(0.6 \%)$ & $3(0.6 \%)$ & 0 \\
\hline
\end{tabular}

Values are mean (SD) or $\mathrm{n}(\%)$.

*Defined as close relative with AMI, angina or cardiac death before the age of 55 years.

ACS, acute coronary syndrome; ADP, accelerated diagnostic protocol; ADP inhibitor, adenosine diphosphate receptor inhibitor; ARB blocker, angiotensin II receptor blocker; CABG, coronary artery bypass graft; MACE, major adverse cardiovascular events; $\mathrm{PCl}$, percutaneous coronary intervention; TIA, transitory ischaemic attack.

the HEART score had an NPV for index visit AMI of 99.2\%. As the average ED ACS prevalence is $13 \%-14 \%,{ }^{16}$ and as all strategies had LRs of 0.02-0.09, a low miss rate should be attainable in most settings, since even with a higher pretest probability, such low LRs would result in a low post-test probability.

Although the HEART 0-hour/1-hour Pathway seemed to miss fewer patients with 30-day MACE than the EDACS 0-hour/1-hour ADP, this difference was not statistically significant and hence might be due to chance alone. It should be noted however, that only the HEART 0-hour/1-hour Pathway had both a sensitivity $>99 \%$ and an NPV of $\geq 99.5 \%$ for both 30 -day MACE and AMI.

Previous studies of the HEART Pathway have primarily used 0 -hour and 3-hour troponin testing and not high sensitivity Tn

Table 2 Diagnostic accuracy of the algorithms for ruling out 30-day MACE

\begin{tabular}{|c|c|c|c|c|}
\hline & $\begin{array}{l}\text { Sensitivity \% } \\
(95 \% \mathrm{Cl})\end{array}$ & $\begin{array}{l}\text { Specificity \% } \\
(95 \% \mathrm{CI})\end{array}$ & $\begin{array}{l}\text { NPV } \\
(95 \% \mathrm{CI})\end{array}$ & $\begin{array}{l}\text { LR- } \\
(95 \% \mathrm{Cl})\end{array}$ \\
\hline \multicolumn{5}{|l|}{ 30-day MACE } \\
\hline HEART score & 94.8 (89.1 to 98.1$)$ & 60.2 (56.7 to 63.5$)$ & 98.8 (97.4 to 99.5$)$ & $0.09(0.04$ to 0.19$)$ \\
\hline HEART 0-hour/1-hour Pathway & 99.1 (95.3 to 99.9) & 56.7 (53.3 to 60.2$)$ & $99.8(98.5$ to 100.0$)$ & $0.02(0.00$ to 0.11$)$ \\
\hline EDACS 0-hour/1-hour ADP & 96.7 (91.4 to 99.1$)$ & 56.1 (52.7 to 59.6$)$ & 99.1 (97.8 to 99.7) & 0.06 (0.02 to 0.16$)$ \\
\hline \multicolumn{5}{|l|}{ Index visit MI } \\
\hline HEART score & 94.7 (86.9 to 98.5$)$ & 57.5 (54.2 to 60.9$)$ & 99.2 (98.0 to 99.7$)$ & $0.09(0.04$ to 0.24$)$ \\
\hline HEART 0-hour/1-hour Pathway & $100.0(95.2$ to 100.0$)$ & 54.2 (50.8 to 57.5$)$ & $100.0(99.2$ to 100$)$ & $0.00(0-\mathrm{NaN})$ \\
\hline EDACS 0-hour/1-hour-ADP & 98.7 (92.8 to 100.0$)$ & 53.8 (50.4 to 57.2$)$ & 99.8 (98.5 to 100.0$)$ & $0.02(0.00$ to 0.17$)$ \\
\hline
\end{tabular}

ADP, accelerated diagnostic protocol; EDACS, Emergency Department Assessment of Chest Pain Score; HEART, History, ECG, Age, Risk Factors and Troponin; LR-, negative likelihood ratio; MACE, major adverse cardiac events; NPV, negative predictive value. 
assays. ${ }^{9}$ In a recent meta-analysis this approach was shown to have an NPV of $99.2 \%$ and the authors stated that using hs-cTn could be beneficial. ${ }^{17}$ Our results suggest that the HEART Pathway can be safely combined with hs-cTn 0-hour/1-hour testing, potentially enabling faster rule-out. Our use of hs-cTnT also seemed to increase the NPV of the original HEART score, which has an NPV of only $97 \%-98 \%$ for MACE $^{18}$ with previous generation Tn assays. Our results are also in line with a metaanalysis reporting that the original HEART score combined with hs-cTnT had an NPV for 30-day MACE of 98.8\% and an LRof $0.09 .^{17}$

The EDACS-ADP has previously been evaluated in studies using 0 -hour and 2-hour troponin testing ${ }^{10}$ and shown to be able to safely rule-out 30-day MACE. Our study is the first to evaluate the EDACS-ADP using hs-cTnT and 0-hour/1-hour testing, and it confirms and extends previous findings that EDACS-ADP can safely rule-out adverse cardiac events in ED chest pain patients.

As opposed to our 0-hour/1-hour hs-cTnT protocol, Greenslade et $a l^{19}$ studied the diagnostic accuracy of the HEART Pathway and EDACS when combined with 0 -hour/2-hour hs-cTnI testing, and their results were quite similar to ours. A HEART score $<4$ with 0 -hour and 2 -hour hs-cTnI $<18 \mathrm{ng} / \mathrm{L}$ identified the same proportion of patients for rule-out $(49.8 \%)$ as in our study, with an NPV for 30-day AMI of 99.8\%. An EDACS score <16, nonischaemic ECG and 0-hour/2-hour hs-cTnI $<18 \mathrm{ng} / \mathrm{L}$ identified a slightly larger proportion of patients as low risk $(62.5 \%$ vs our $49.6 \%$ ), with a sensitivity of $92.1 \%$ and NPV of $99.8 \%$ in comparison to our EDACS 0-hour/1-hour ADP where the sensitivity was $98.7 \%$ and NPV $99.8 \%$.

The use of 0-hour/1-hour hs-cTnT testing alone has been shown to be able to safely rule-out $\mathrm{AMI},{ }^{7}$ but not 30-day MACE including UA. ${ }^{720}$ This indicates that 0 -hour/1-hour testing should be combined with some form of clinical assessment. We found that the risk of MACE including UA was very low in patients assessed as low risk by all three strategies, which probably makes further testing (with eg, imaging or stress tests) for ACS unnecessary. This confirms previous findings with the HEART Pathway, ${ }^{9}$ but is a novel finding with EDACS-ADP. Several other risk scores for chest pain evaluation such as the Vancouver chest pain score have also been evaluated for an outcome including UA, ${ }^{21}$ and have identified $<25 \%$ of patients for rule-out with varying miss rates. ${ }^{21}$ In a previous study, we found that a combination algorithm including patient history, ECG and 0-hour/1-hour hs-cTnT safely ruled out 30-day MACE including UA in $60 \%$ of ED chest pain patients. ${ }^{7}$ A potential disadvantage with both this algorithm and the HEART score is that they incorporate a subjective history assessment, which might make some clinicians reluctant to use them. Both the HEART score and ED physicians' assessment of chest pain history have been shown to have kappa values of only $\geq 0.6$ for interobserver agreement. ${ }^{9}$ Although this is classified as 'good', it implies assessment variation between physicians which could affect how patients are risk stratified.

The UK National Institute for Health and Care Excellence (NICE) guidelines recommend that scores like HEART and EDACS can be used for rule-out together with a single hs-cTnT $<5 \mathrm{ng} / \mathrm{L},{ }^{22}$ regardless of the time of chest pain onset. Although based on a limited number of events, the similar diagnostic accuracy in patients presenting $\leq 3$ hours versus $>3$ hours after chest pain onset support that a single hs-cTnT $<5 \mathrm{ng} / \mathrm{L}$ together with the HEART score or the EDACS-ADP can be used for rule-out both in early and later presenters. These results are also in line with the findings of the LoDED trial. ${ }^{23}$

Our study adds to the growing body of literature supporting the safety of the HEART Pathway and EDACS-ADP. ${ }^{9} 10171824$
More importantly, it shows that these strategies can be combined with guideline-recommended 0 -hour/1-hour hs-cTnT testing, potentially enabling faster patient disposition. Our data also suggest that if the original HEART score is used, this should incorporate hs-cTnT to lower MACE miss rates.

\section{LIMITATIONS}

We performed a single centre study which limits the generalisability of our findings. However, our AMI/MACE prevalence was similar to that in previous studies on ED chest pain patients, ${ }^{2}$ and to the reported average ED prevalence. ${ }^{16}$ Furthermore, all strategies had an LR $-<0.1$ for 30-day MACE, lowering the risk significantly and allowing safe use of the algorithms even in settings with a higher AMI/MACE prevalence.

We included patients only during the day and not during the night or weekends, but we have previously shown that the patient population during nights and weekends is similar to the included patients with regard to age, sex and prevalence of AMI. ${ }^{7}$ Studies with 24 hours patient inclusion at our ED ${ }^{25}$ show similar prevalence for AMI and UA, which indicates that the population in the present study was representative of all our ED chest pain patients.

We excluded patients with missing data, which might cause selection bias. However, since the baseline characteristics between included and excluded patients were similar (online supplemental appendix table 4), the risk for such bias is probably small.

In our comparison between patients presenting $\leq 3$ hours versus $>3$ hours after chest pain onset, there was no statistical difference in the diagnostic accuracy of the algorithms (online supplemental appendix table 5). However, this was a post-hoc analysis and CIs were wide and these results should therefore only be seen as hypothesis generating.

In contrast to previous studies on EDACS, patients with ongoing chest pain or symptoms compatible with crescendo angina were not excluded, and this might have affected the results of the EDACS-ADP.

One could argue whether UA should be included in a MACE outcome. We however believe this is motivated since it is common, ${ }^{12}$ carries a significant risk of cardiac events ${ }^{12}$ and changes management. ${ }^{5}$ We also believe that since treatment for UA may prevent other adverse events such as AMI and death, leaving out UA in the outcome may introduce bias in an observational study such as ours.

This was a secondary analysis of data from a prospective observational study, and definitive conclusions regarding clinical safety are therefore not possible. However, one large before and after implementation study has suggested that risk scores such as EDACS are effective strategies for chest pain assessment and that their use can safely increase early discharges. ${ }^{10}$ Before clinical implementation, our results should however be validated in other settings, preferably in prospective multicenter studies.

\section{CONCLUSION}

The present results indicate that the combination of the HEART Pathway or the EDACS-ADP with a 0-hour/1-hour hs-cTnT protocol allows safe and early rule-out in a large proportion of ED chest pain patients. In our cohort, the original HEART score had a slightly poorer diagnostic accuracy. Prospective implementation studies analysing the effectiveness of these two algorithms in routine care are warranted.

Acknowledgements We thank the patients for their participation, and the research assistants and the emergency department staff for their kind help. 
Contributors All authors have read and approved the final manuscript and meet the criteria for authorship as established by the ICMJE.

Funding The study was supported by an ALF research grant (grant no. 2018-Projekt0152) at Skåne University Hospital and by a grant from Region Skåne (grant no. REGSKANE-814271). These are grants from the Swedish government and the regional council. There was no industry involvement. This study was part of the AIR Lund (Artificially Intelligent use of Registers at Lund University) research environment, and received funding from the Swedish Research Council (VR; grant no. 2019-00198). Further funding was provided by a grant from the Heart-Lung fund (Hjärt-Lungfonden, grant no. 20180173). Funding organisations had no role in the planning, design, or conduct of the study, collection, analysis or interpretation of data, or preparation, review or approval of the manuscript.

Competing interests None declared.

Patient consent for publication Not required.

Ethics approval This study was approved by the Regional Ethics Review Board of Lund, $\mathrm{Nr}$ 2013/197+2015/76. All included patients consented in writing.

Provenance and peer review Not commissioned; externally peer reviewed. Data availability statement Data are available upon reasonable request.

Supplemental material This content has been supplied by the author(s). It has not been vetted by BMJ Publishing Group Limited (BMJ) and may not have been peer-reviewed. Any opinions or recommendations discussed are solely those of the author(s) and are not endorsed by BMJ. BMJ disclaims all liability and responsibility arising from any reliance placed on the content. Where the content includes any translated material, BMJ does not warrant the accuracy and reliability of the translations (including but not limited to local regulations, clinical guidelines, terminology, drug names and drug dosages), and is not responsible for any error and/or omissions arising from translation and adaptation or otherwise.

\section{ORCID iD}

Tsvetelina Nilsson http://orcid.org/0000-0002-6384-0504

\section{REFERENCES}

1 Stepinska J, Lettino M, Ahrens I, et al. Diagnosis and risk stratification of chest pain patients in the emergency department: focus on acute coronary syndromes. A position paper of the acute cardiovascular care association. Eur Heart J Acute Cardiovasc Care 2020;9:76-89.

2 Cullen L, Greenslade J, Merollini K, et al. Cost and outcomes of assessing patients with chest pain in an Australian emergency department. Med J Aust 2015;202:427-32.

3 Nilsson T, Lundberg G, Larsson D, et al. Emergency department chest pain patients with or without ongoing pain: characteristics, outcome, and diagnostic value of the electrocardiogram. J Emerg Med 2020;58:874-81.

4 Eriksson D, Khoshnood A, Larsson D, et al. Diagnostic Accuracy of History and Physical Examination for Predicting Major Adverse Cardiac Events Within 30 Days in Patients With Acute Chest Pain. J Emerg Med 2019. doi:10.1016/j.jemermed.2019.09.044. [Epub ahead of print: 25 Nov 2019].

5 Collet J-P, Thiele H, Barbato E. 2020 ESC guidelines for the management of acute coronary syndromes in patients presenting without persistent ST-segment elevation. Eur Heart J 2020;ehaa575.
6 McCord J, Cabrera R, Lindahl B, et al. Prognostic utility of a modified heart score in chest pain patients in the emergency department. Circ Cardiovasc Qual Outcomes 2017;10.

7 Mokhtari A, Borna C, Gilje P, et al. A 1-h Combination Algorithm Allows Fast Rule-Out and Rule-In of Major Adverse Cardiac Events. J Am Coll Cardiol 2016;67:1531-40.

8 Borna C, Kollberg K, Larsson D, et al. The objective core score allows early rule out in acute chest pain patients. Scand Cardiovasc J 2018;52:308-14.

9 Mahler SA, Riley RF, Hiestand BC, et al. The heart pathway randomized trial: identifying emergency department patients with acute chest pain for early discharge. Circ Cardiovasc Qual Outcomes 2015;8:195-203.

10 Than MP, Pickering JW, Dryden JM, et al. ICare-ACS (improving care processes for patients with suspected acute coronary syndrome): a study of Cross-System implementation of a national clinical pathway. Circulation 2018;137:354-63.

11 Sandoval Y, Jaffe AS. Using high-sensitivity cardiac troponin T for acute cardiac care. Am J Med 2017;130:e1351:1358-65.

12 Eggers KM, Jernberg T, Lindahl B. Unstable angina in the era of cardiac troponin assays with improved Sensitivity-A clinical dilemma. Am J Med 2017;130:e1425:1423-30.

13 Cullen L, Than M, Brown AFT, et al. Comprehensive standardized data definitions for acute coronary syndrome research in emergency departments in Australasia. Emerg Med Australas 2010;22:35-55.

14 Kline JA, Johnson CL, Pollack CV, et al. Pretest probability assessment derived from attribute matching. BMC Med Inform Decis Mak 2005;5:26.

15 Pickering JW. The need to improve derivation and description of algorithms to RuleOut patients with possible myocardial infarction. Circulation 2019;139:1351-3.

16 Fanaroff AC, Rymer JA, Goldstein SA, et al. Does this patient with chest pain have acute coronary syndrome?: the rational clinical examination systematic review. JAMA 2015;314:1955-65.

17 Laureano-Phillips J, Robinson RD, Aryal S, et al. Heart score risk stratification of low-risk chest pain patients in the emergency department: a systematic review and meta-analysis. Ann Emerg Med 2019;74:187-203.

18 Van Den Berg P, Body R. The heart score for early rule out of acute coronary syndromes in the emergency department: a systematic review and meta-analysis. Eur Heart J Acute Cardiovasc Care 2018;7:111-9.

19 Greenslade JH, Carlton EW, Van Hise C, et al. Diagnostic accuracy of a new highsensitivity troponin I assay and five accelerated diagnostic pathways for ruling out acute myocardial infarction and acute coronary syndrome. Ann Emerg Med 2018;71:e433:439-51.

20 Mokhtari A, Lindahl B, Schiopu A, et al. A 0-Hour/1-Hour protocol for safe, early discharge of chest pain patients. Acad Emerg Med 2017;24:983-92.

21 Mahler SA, Miller CD, Hollander JE, et al. Identifying patients for early discharge: performance of decision rules among patients with acute chest pain. Int I Cardiol 2013;168:795-802.

22 NICE guidance. Chest pain of recent onset: assessment and diagnosis (update). CG95. London: National Institute for Health and Care Excellence, 2010. https://www.nice. org.uk/guidance/cg95

23 Carlton EW, Ingram J, Taylor H, et al. Limit of detection of troponin discharge strategy versus usual care: randomised controlled trial. Heart 2020;106:1586-94.

24 Khoshnood A, Erlandsson M, Isma N, et al. Diagnostic accuracy of troponin T measured $\geq 6$ h after symptom onset for ruling out myocardial infarction. Scand Cardiovasc J 2020;54:153-61.

25 Mokhtari A, Dryver E, Söderholm M, et al. Diagnostic values of chest pain history, ECG, troponin and clinical gestalt in patients with chest pain and potential acute coronary syndrome assessed in the emergency department. Springerplus 2015;4:219. 